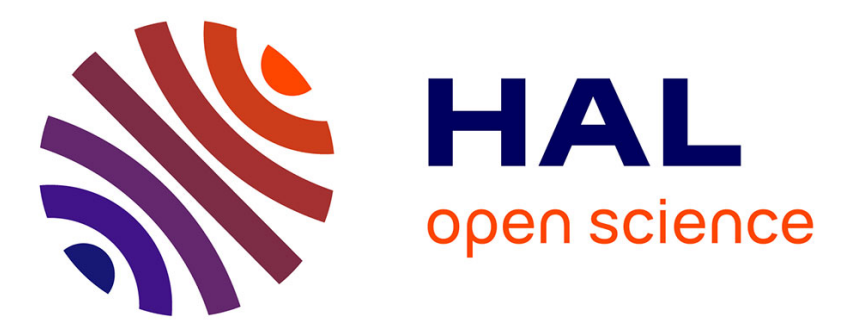

\title{
Coronagraphic wavefront sensing with COFFEE: high spatial-frequency diversity and other news
}

L. Mugnier, J.F. Sauvage, O. Herscovici-Schiller, P. Baudoz, R. Galicher, J.M.

Le Duigou

\section{- To cite this version:}

L. Mugnier, J.F. Sauvage, O. Herscovici-Schiller, P. Baudoz, R. Galicher, et al.. Coronagraphic wavefront sensing with COFFEE: high spatial-frequency diversity and other news. Space Telescopes and Instrumentation 2016: Optical, Infrared, and Millimeter Wave, Jun 2016, EDINBURGH, United Kingdom. hal-01384167

\section{HAL Id: hal-01384167 \\ https://hal.science/hal-01384167}

Submitted on 24 Oct 2016

HAL is a multi-disciplinary open access archive for the deposit and dissemination of scientific research documents, whether they are published or not. The documents may come from teaching and research institutions in France or abroad, or from public or private research centers.
L'archive ouverte pluridisciplinaire HAL, est destinée au dépôt et à la diffusion de documents scientifiques de niveau recherche, publiés ou non, émanant des établissements d'enseignement et de recherche français ou étrangers, des laboratoires publics ou privés. 


COMMUNICATION A CONGRES
Coronagraphic wavefront sensing
with COFFEE:
high spatial-frequency diversity and
other news
L. Mugnier (ONERA), J.-F. Sauvage (ONERA),
O. Herscovici -Schiller (ONERA), P. Baudoz,
R. Galicher, J.-M. Le Duigou
Space Telescopes and Instrumentation 2016: Optical,
Infrared, and Millimeter Wave
Edinburgh, Grande Bretagne
26 juin-1 juillet 2016
TP 2016-651

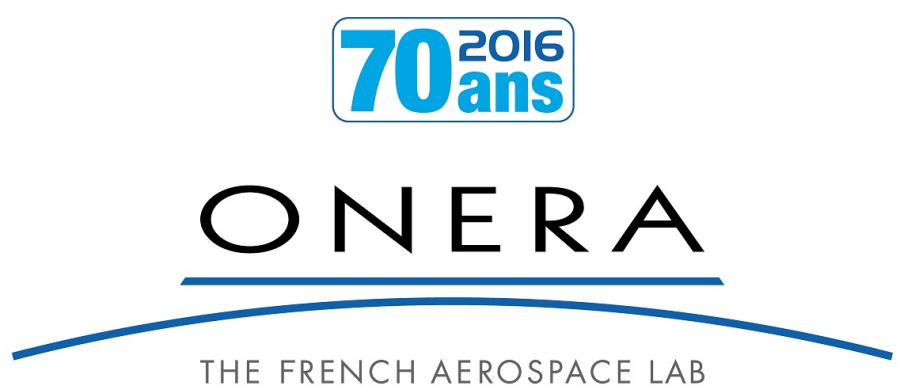





\title{
Coronagraphic wavefront sensing with COFFEE: high spatial-frequency diversity and other news
}

\author{
L. M. Mugnier ${ }^{\mathrm{a}}$, J.-F. Sauvage ${ }^{\mathrm{a}, \mathrm{b}}$, O. Herscovici-Schiller ${ }^{\mathrm{a}}$, P. Baudoz ${ }^{\mathrm{c}}$, R. Galicher ${ }^{\mathrm{c}}$, and J.-M. \\ Le Duigou ${ }^{\mathrm{d}}$ \\ ${ }^{\mathrm{a} O N E R A ~-~ T h e ~ F r e n c h ~ A e r o s p a c e ~ L a b, ~ F-92322 ~ C h a ̂ t i l l o n, ~ F r a n c e ~}$ \\ ${ }^{\mathrm{b}}$ Laboratoire d'Astrophysique de Marseille, UMR 7326, Marseille, France \\ ${ }^{\mathrm{c}}$ Observatoire de Paris and Lab. d'Etudes Spatiales et d'Instrumentation en Astrophysique, Meudon, France \\ ${ }^{\mathrm{d}}$ Centre National d'Etudes Spatiales, Toulouse, France
}

\begin{abstract}
The final performance of current and future instruments dedicated to exoplanet detection and characterization is limited by intensity residuals in the scientific image plane, which originate in uncorrected optical aberrations. In order to reach very high contrasts, these aberrations needs to be compensated for. We have proposed a focalplane wave-font sensor called COFFEE (for COronagraphic Focal-plane wave-Front Estimation for Exoplanet detection), which consists in an extension of conventional phase diversity to a coronagraphic system. In this communication, we study the extension of COFFEE to the joint estimation of the phase and the amplitude in the context of space-based coronagraphic instruments: we optimize the diversity phase in order to minimize the reconstruction error; we also propose and optimize a novel low-amplitude high-frequency diversity that should allow the phase-diverse images to still be used for science. Lastly, we perform a first experimental validation of COFFEE in the very high, space-like contrast conditions of the THD bench and show that COFFEE is able to distinguish between phase and amplitude aberrations.
\end{abstract}

Keywords: Wavefront sensing, phase diversity, phase retrieval, high contrast imaging, inverse problems, exoplanets.

\section{INTRODUCTION}

The final performance of current and future instruments dedicated to exoplanet detection and characterization (such as SPHERE on the VLT, GPI on Gemini North or future ground or space-based instruments) is limited by intensity residuals in the scientific image plane, which originate in uncorrected optical aberrations. The main contribution to these residuals (after correction of turbulence by Adaptive Optics for ground-based instruments) comes from the quasi-static aberrations introduced upstream of the coronagraph, which create long-lived speckles in the detector plane that can easily be mistaken for a planet. In order to reach very high contrasts such as the ones required to image earth-like planets, these aberrations must be compensated for. We have recently developed a dedicated focal-plane wave-font sensor called COFFEE (for COronagraphic Focal-plane wave-Front Estimation for Exoplanet detection), which consists in an extension of conventional phase diversity to a coronagraphic system: aberrations both upstream and downstream of the coronagraph are estimated using two coronagraphic focal-plane images, recorded from the scientific camera itself, without any differential aberration. ${ }^{1,2}$ Such a system has been successfully validated on the SPHERE instrument, where COFFEE's estimation has been used to compensate for the phase aberration upstream of the coronagraph, leading to a contrast optimization in the whole focal plane area controlled by the AO loop. ${ }^{3}$ For planet-finder instruments that will aim at detecting and characterizing Earth-like planets from the ground or from space, several orders of magnitude in contrast must still be gained. This requires measuring and compensating not only the phase but also the amplitude aberrations (inhomogeneous intensity in the pupil, due to Fresnel propagation). In this communication, we study the extension of COFFEE to perform a simultaneous estimation of both phase and amplitude aberration from three or more focal plane images. We optimize the diversity phase in order to minimize the reconstruction

E-mail: mugnier@onera.fr

Space Telescopes and Instrumentation 2016: Optical, Infrared, and Millimeter Wave, edited by Howard A. MacEwen, Giovanni G. Fazio, Makenzie Lystrup, Proc. of SPIE Vol. 9904, 99043A · ( 2016 SPIE · CCC code: 0277-786X/16/\$18 - doi: 10.1117/12.2233726

Proc. of SPIE Vol. $990499043 A-1$ 
error. We also propose and optimize a novel low-amplitude high-frequency diversity that should allow the phasediverse images to still be used for science. Lastly, we perform a first experimental validation of COFFEE in the very high, space-like contrast conditions of the THD bench of Observatoire de Paris and show that COFFEE is able to distinguish between phase and amplitude aberrations.

COFFEE has the advantage, with respect to approaches such as speckle nulling ${ }^{4}$ or the Electric Field Conjugation (EFC), ${ }^{5}$ that it does not assume small aberrations and does not perform a Taylor expansion of the complex field. COFFEE is thus likely to lead to a correction that converges faster and needs less image acquisitions. Compared to the SCC approach, ${ }^{6}$ COFFEE needs more images but does not require any modification of the imaging system, and can thus be integrated very easily into any high-contrast imaging system. Compared to ZELDA, ${ }^{7}$ COFFEE has the advantage that it uses coronagraphic images of the scientific detector and is thus immune to Non-Common-Path Aberrations (NCPA).

\section{MEASURING AMPLITUDE AND PHASE WITH COFFEE}

\subsection{Image formation model}

We consider a coronagraphic imaging system observing a point source. Let $\exp i . \phi_{u}+\boldsymbol{\xi}_{u}$ be the complex field to be estimated in a pupil plane upstream of the coronagraph, and $\phi_{d}$ the phase aberrations in a pupil plane downstream of the coronagraph. We denote by $\boldsymbol{h}_{c}\left(\boldsymbol{\phi}_{u}, \boldsymbol{\xi}_{u}, \boldsymbol{\phi}_{d}\right)$ the model of the coronagraphic PSF as a function of $\phi_{u}, \boldsymbol{\xi}_{u}, \phi_{d}$. We assume that we record three coronagraphic images in the focal plane: one focused image $\boldsymbol{i}_{c}^{\text {foc }}$ and two diversity images $\phi_{\mathrm{div}_{1}}$ and $\phi_{\mathrm{div}_{2}}$, differing from the focused image by a known diversity, $\phi_{\mathrm{div}_{1}}$ and $\phi_{\mathrm{div}_{2}}$ respectively. These images can be written as:

$$
\begin{aligned}
\boldsymbol{i}_{c}^{\mathrm{foc}} & =\alpha_{\mathrm{foc}} \boldsymbol{h}_{\mathrm{det}} \star \boldsymbol{h}_{c}\left(\boldsymbol{\phi}_{u}, \boldsymbol{\xi}_{u}, \boldsymbol{\phi}_{d}\right)+n_{\mathrm{foc}}+\beta_{\mathrm{foc}} \\
\boldsymbol{i}_{c}^{\mathrm{div} 1} & =\alpha_{\mathrm{div} 1} \boldsymbol{h}_{\mathrm{det}} \star \boldsymbol{h}_{c}\left(\boldsymbol{\phi}_{u}+\boldsymbol{\phi}_{\mathrm{div} 1}, \boldsymbol{\xi}_{u}, \boldsymbol{\phi}_{d}\right)+n_{\mathrm{div} 1}+\beta_{\mathrm{div} 1} . \\
\boldsymbol{i}_{c}^{\mathrm{div} 2} & =\alpha_{\mathrm{div} 2} \boldsymbol{h}_{\mathrm{det}} \star \boldsymbol{h}_{c}\left(\boldsymbol{\phi}_{u}+\boldsymbol{\phi}_{\mathrm{div} 2}, \boldsymbol{\xi}_{u}, \boldsymbol{\phi}_{d}\right)+n_{\mathrm{div} 2}+\beta_{\mathrm{div} 2},
\end{aligned}
$$

where $\alpha_{p}$ is the flux of the star $(p \in\{$ foc, div1, div2 $\}), \beta_{p}$ represents a constant residual background in image $\boldsymbol{i}_{c}^{p}$, $\boldsymbol{h}_{\mathrm{det}}$ is the detector PSF, and $\boldsymbol{n}_{p}$ denotes the measurement noise. This noise is usually a combination of photon and detector noises, which can be well approximated by a white Gaussian inhomogeneous noise for the flux levels at hand in high contrast imaging. Its variance is then the sum of the variances of the photon noise $\sigma_{\operatorname{det}_{p}}^{2}$ and of the detector noise $\boldsymbol{\sigma}_{\text {phot }_{p}}^{2}$ :

$$
\boldsymbol{\sigma}_{n_{p}}^{2}[k]=\sigma_{\operatorname{det}_{p}}^{2}+\boldsymbol{\sigma}_{\text {phot }_{p}}^{2}[k]
$$

where $k$ is the current pixel index. In this equation, $\sigma_{\operatorname{det}_{p}}$ is a scalar determined by the calibration of the detector, and the variance map $\boldsymbol{\sigma}_{\text {phot }_{p}}^{2}[k]$ can be estimated simply from the image $\boldsymbol{i}_{c}^{p}$ itself $^{8}$ :

$$
\boldsymbol{\sigma}_{\text {phot }}^{2}[k]=\max \left(i_{c}^{p}[k], 0\right)
$$

\subsection{MAP metric for phase and amplitude reconstruction}

The COFFEE method is based on the optimization of a Maximum A Posteriori (MAP) metric. It consists in searching for the upstream phase and amplitude aberrations $\hat{\boldsymbol{\phi}}_{u}$ and $\hat{\boldsymbol{\xi}}_{u}$, the downstream phase aberrations $\hat{\boldsymbol{\phi}}_{d}$, as well as the fluxes $\hat{\boldsymbol{\alpha}}=\left[\hat{\alpha}_{\mathrm{foc}}, \hat{\alpha}_{\mathrm{div} 1}, \hat{\alpha}_{\mathrm{div} 2}\right]$ and the background values $\hat{\boldsymbol{\beta}}=\left[\hat{\beta}_{\mathrm{foc}}, \hat{\beta}_{\mathrm{div} 1}, \hat{\beta}_{\mathrm{div} 2}\right]$ that maximize the posterior likelihood $p\left(\boldsymbol{\alpha}, \boldsymbol{\beta}, \boldsymbol{\phi}_{u}, \boldsymbol{\xi}_{u}, \boldsymbol{\phi}_{d} \mid \boldsymbol{i}_{c}^{\text {foc }}, \boldsymbol{i}_{c}^{\text {div1 }}, \boldsymbol{i}_{c}^{\text {div2 }}\right)$. In practice, the solution is obtained by the minimization of the neg-log-likelihood metric $J$ defined as the opposite of the logarithm of this posterior likelihood, i.e.,:

$$
\left(\hat{\boldsymbol{\alpha}}, \hat{\boldsymbol{\beta}}, \hat{\boldsymbol{\phi}}_{u}, \hat{\boldsymbol{\xi}}_{u}, \hat{\boldsymbol{\phi}}_{d}\right)=\underset{\boldsymbol{\alpha}, \boldsymbol{\beta}, \boldsymbol{\phi}_{u}, \boldsymbol{\xi}_{u}, \phi_{d}}{\arg \min } J\left(\boldsymbol{\alpha}, \boldsymbol{\beta}, \boldsymbol{\phi}_{u}, \boldsymbol{\xi}_{u}, \boldsymbol{\phi}_{d}\right),
$$


with:

$$
\begin{aligned}
J\left(\boldsymbol{\alpha}, \boldsymbol{\beta}, \boldsymbol{\phi}_{u}, \boldsymbol{\xi}_{u}, \phi_{d}\right)= & \frac{1}{2}\left\|\frac{\boldsymbol{i}_{c}^{\mathrm{foc}}-\left[\alpha_{\mathrm{foc}} \boldsymbol{h}_{\mathrm{det}} \star \boldsymbol{h}_{c}\left(\boldsymbol{\phi}_{u}, \boldsymbol{\xi}_{u}, \boldsymbol{\phi}_{d}\right)+\beta_{\mathrm{foc}}\right]}{\boldsymbol{\sigma}_{n_{\mathrm{foc}}}}\right\|^{2} \\
& +\frac{1}{2}\left\|\frac{\boldsymbol{i}_{c}^{\mathrm{div} 1}-\left[\alpha_{\mathrm{div} 1} \boldsymbol{h}_{\mathrm{det}} \star \boldsymbol{h}_{c}\left(\boldsymbol{\phi}_{u}+\boldsymbol{\phi}_{\mathrm{div} 1}, \boldsymbol{\xi}_{u}, \boldsymbol{\phi}_{d}\right)+\beta_{\mathrm{div} 1}\right]}{\boldsymbol{\sigma}_{n_{\mathrm{div} 1}}}\right\|^{2} \\
& +\frac{1}{2}\left\|\frac{\boldsymbol{i}_{c}^{\mathrm{div} 2}-\left[\alpha_{\mathrm{div} 2} \boldsymbol{h}_{\mathrm{det}} \star \boldsymbol{h}_{c}\left(\boldsymbol{\phi}_{u}+\boldsymbol{\phi}_{\mathrm{div} 2}, \boldsymbol{\xi}_{u}, \boldsymbol{\phi}_{d}\right)+\beta_{\mathrm{div} 2}\right]}{\boldsymbol{\sigma}_{n_{\mathrm{div} 2}}}\right\|^{2} \\
& +\mathcal{R}\left(\boldsymbol{\phi}_{u}\right)+\mathcal{R}\left(\boldsymbol{\xi}_{u}\right)+\mathcal{R}\left(\boldsymbol{\phi}_{d}\right),
\end{aligned}
$$

where $\mathcal{R}\left(\phi_{q}\right)$ represents the regularization term on the phase aberration $\phi_{q}$, with $q \in[u, d]$, and $\mathcal{R}\left(\boldsymbol{\xi}_{u}\right)$ represents the regularization term on the amplitude aberrations $\boldsymbol{\xi}_{u}$. The minimization of metric $J$ (Equation 5 ) is performed by means of the VMLM-B method, ${ }^{9}$ and relies on the analytical expressions of gradients $\partial J / \partial \phi_{u}, \partial J / \partial \boldsymbol{\xi}_{u}$ $\partial J / \partial \phi_{d}, \partial J / \partial \boldsymbol{\alpha}$ and $\partial J / \partial \boldsymbol{\beta}$.

\subsection{Choice of appropriate basis and regularization for the aberrations}

The use of a pixel basis for the phase reconstruction is required for COFFEE to estimate high order aberrations. However, this leads to a large number of unknowns, which in turn calls for a regularization metric in order to reduce the noise sensitivity. We chose a regularization metric that is based on the available a priori knowledge on the quasi-static aberrations. Indeed, they can be reasonably assumed to be Gaussian, homogeneous and thus endowed with a power spectral density (PSD). In this communication, we use the regularizations in $1 / f^{2}$ and $1 / f^{4}$ whose implementations are described in Ref. 2 , both for the phase and for the amplitude regularizations.

The choice of the diversity phases $\phi_{\mathrm{div} 1}$ and $\phi_{\mathrm{div} 2}$ is a degree of freedom of the method, and is studied in the following sections.

\section{OPTIMIZING THE DIVERSITY FOR COFFEE}

In this section, we assess the quality of the reconstruction of phase and amplitude aberrations with COFFEE. We consider a set of three coronagraphic images, one at the best focus, and two additional images with known diversity phases $\phi_{d}^{1}$ and $\phi_{d}^{2}$. The imaging wavelength is $1600 \mathrm{~nm}$, representative of a science camera in $\mathrm{H}$ band. Additionally, because we consider a calibration context, we consider that the images are high flux $\left(10^{9}\right.$ photon$\mathrm{s} /$ image) and that the detector is low noise (3 electrons/pixel). The aberrations to be measured have a RMS value of about $0.15 \mathrm{rad}(40 \mathrm{~nm})$.

We first illustrate the use of COFFEE with simulations that use the conventional defocus diversity, with $125 \mathrm{~nm}$ RMS and $500 \mathrm{~nm}$ RMS diversity. The coronagraphic images and the images reconstructed by COFFEE are shown on Fig. 1. These images lead to nanometric reconstruction errors shown on Fig. 2.

Next, we wish to optimize the diversity phases. We do this in two steps: we begin by optimizing the focus diversity in the simplified setting where we have only two images and search only for the upstream and downstream phases. Figure 3 shows the reconstruction error as a function of the amplitude of the diversity. We see that a diversity of at least $10 \mathrm{~nm}$ is required to estimate the upstream phase with a nanometric error, and that a much larger diversity, of more than $125 \mathrm{~nm}$, is required if one wants a nanometric error both on the upstream and downstream phases, which is consistent with the results obtained in Ref. 2. We then set the first diversity to $10 \mathrm{~nm}$, and explore the influence of the second diversity on the reconstruction error in the setting where we have three images and search for the upstream phase and amplitude aberrations as well as the downstream aberrations. The results are shown on Figure 4. 


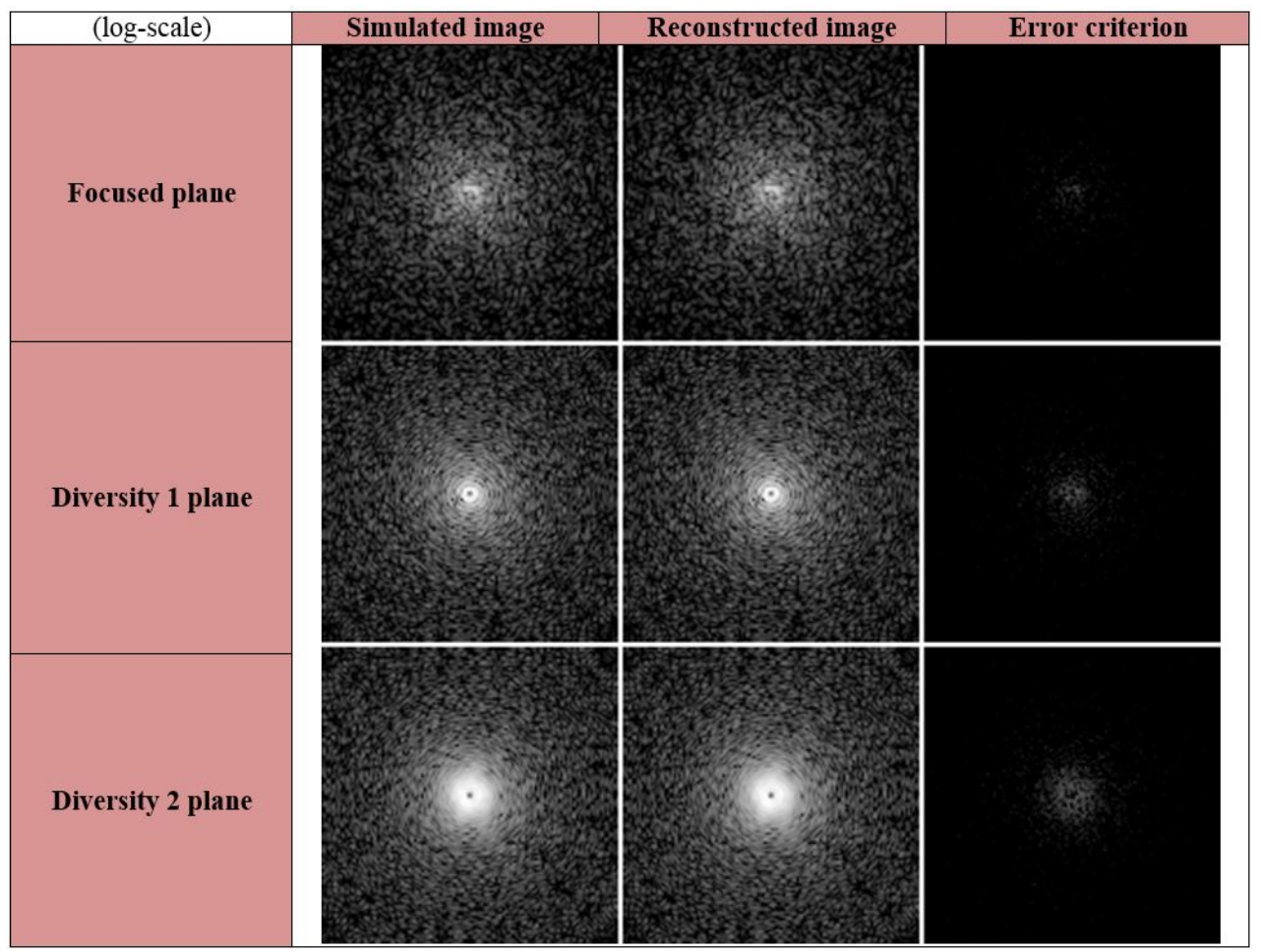

Figure 1. Simulated images (left column), images reconstructed by COFFEE (middle column), and difference (right column), for a focus diversity. All images are shown in log scale.

\begin{tabular}{|c|c|c|c|}
\hline (Linear scale) & Simulated phase & Reconstructed phase & Reconstruction error \\
\hline \multicolumn{4}{|c|}{$\begin{array}{c}\text { Upstream aberration } \\
\text { phase map }\end{array}$} \\
\hline WFE [nm] & 50 & 50 & 0.3 \\
\hline \multicolumn{4}{|c|}{$\begin{array}{c}\text { Downstream } \\
\text { aberration phase map }\end{array}$} \\
\hline WFE [nm] & 20 & 20 & 0.5 \\
\hline
\end{tabular}

Figure 2. Simulated aberrations (left column), aberrations reconstructed by COFFEE (middle column), and difference (right column), for the upstream aberrations (top) and the downstream ones (bottom). The simulated aberrations are reconstructed with a nanometric RMS precision. 


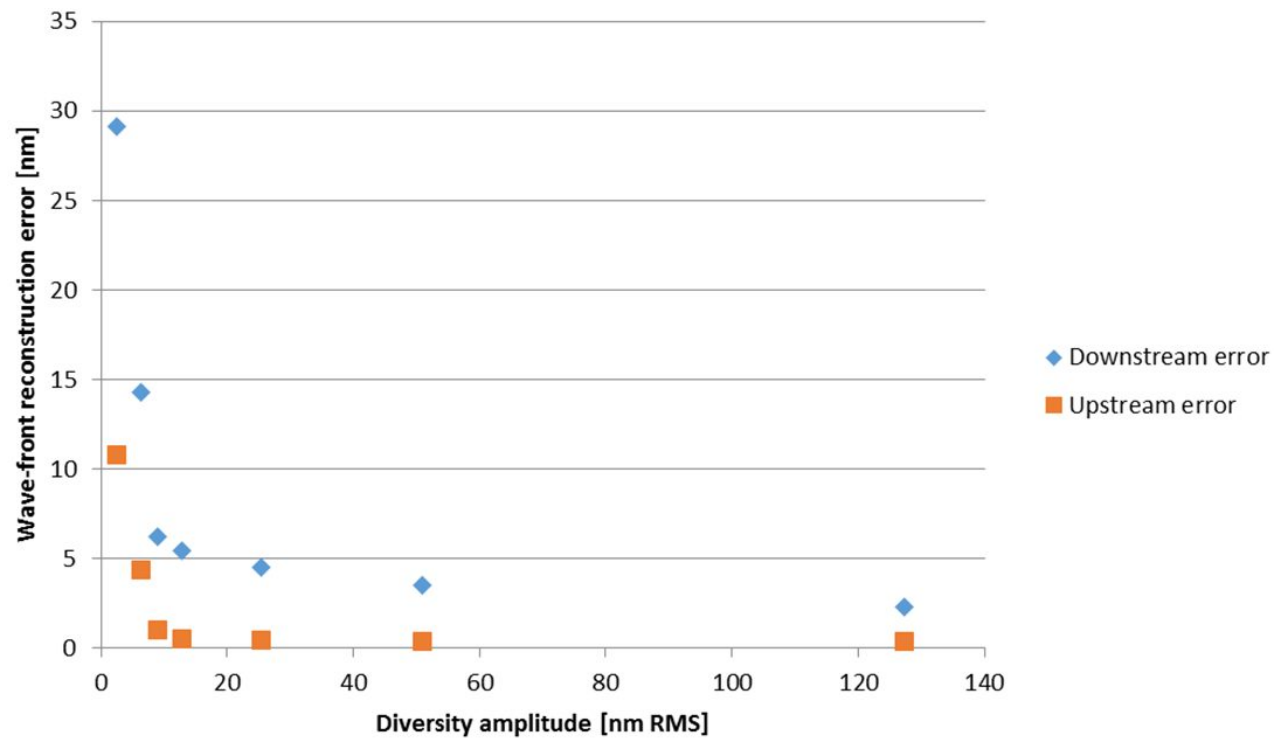

Figure 3. Optimization of the diversity phase. The diversity is a defocus, and only the upstream and downstream phases are reconstructed, using the focused image and one diversity image.

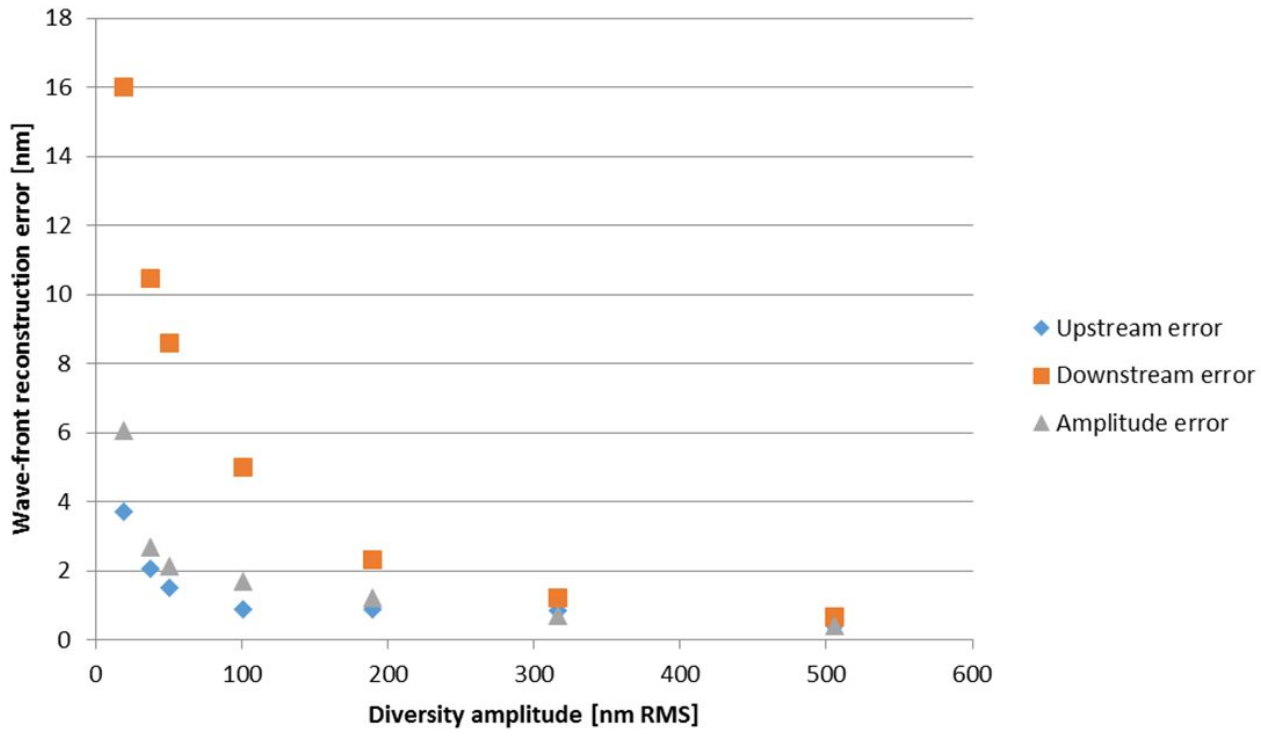

Figure 4. Optimization of the diversity phase for the second diversity image. The diversity of the first diversity image is a defocus, of amplitude $0.05 \mathrm{rad}(12 \mathrm{~nm})$, that of the second diversity image is a defocus of increasing amplitude. Upstream and downstream phases as well as upstream amplitude are reconstructed, using the focused image and two diversity images. 


\section{EXPLORING A NOVEL LOW-AMPLITUDE AND HIGH-FREQUENCY DIVERSITY}

The usual diversity phases used in classical (i.e., non coronagraphic) phase diversity, ${ }^{10}$ in classical phase retrieval (see, e.g.,, Ref. 11), and in $\mathrm{COFFEE}^{1,3,12}$ are of low order and typically consist of defocus and/or astigmatism. For coronagraphic imaging, this has the unfortunate effect that it renders the diversity images unusable for science, as it brings a substancial amount of additional light onto the detector.

That is why we explore below the possibility to use a high-spatial-frequency and low-amplitude diversity, with the aim of keeping most of the corrected zone of the focal plane useable for science. The first diversity we choose is a waffle mode, made of the product of two orthogonal cosine waves (one along $\mathrm{x}$ and one along y), having 20 cycles in the aperture. The second diversity is the same as the first one but rotated by $45^{\circ}$. The amplitude aberrations to be estimated are deduced from a pupil intensity measurement made on the THD bench at Observatoire de Meudon. ${ }^{13}$ The phase aberrations are drawn with a $1 / f^{2.4}$ power law which is also representative of aberrations on this bench.

Figure 5 shows an example of simulated and reconstructed images, and the difference between the two, with the proposed waffle diversity. The good fit between the two sets of images suggests that the optimization went well, and indeed the reconstruction errors are quite small, as shown on Fig. 6.

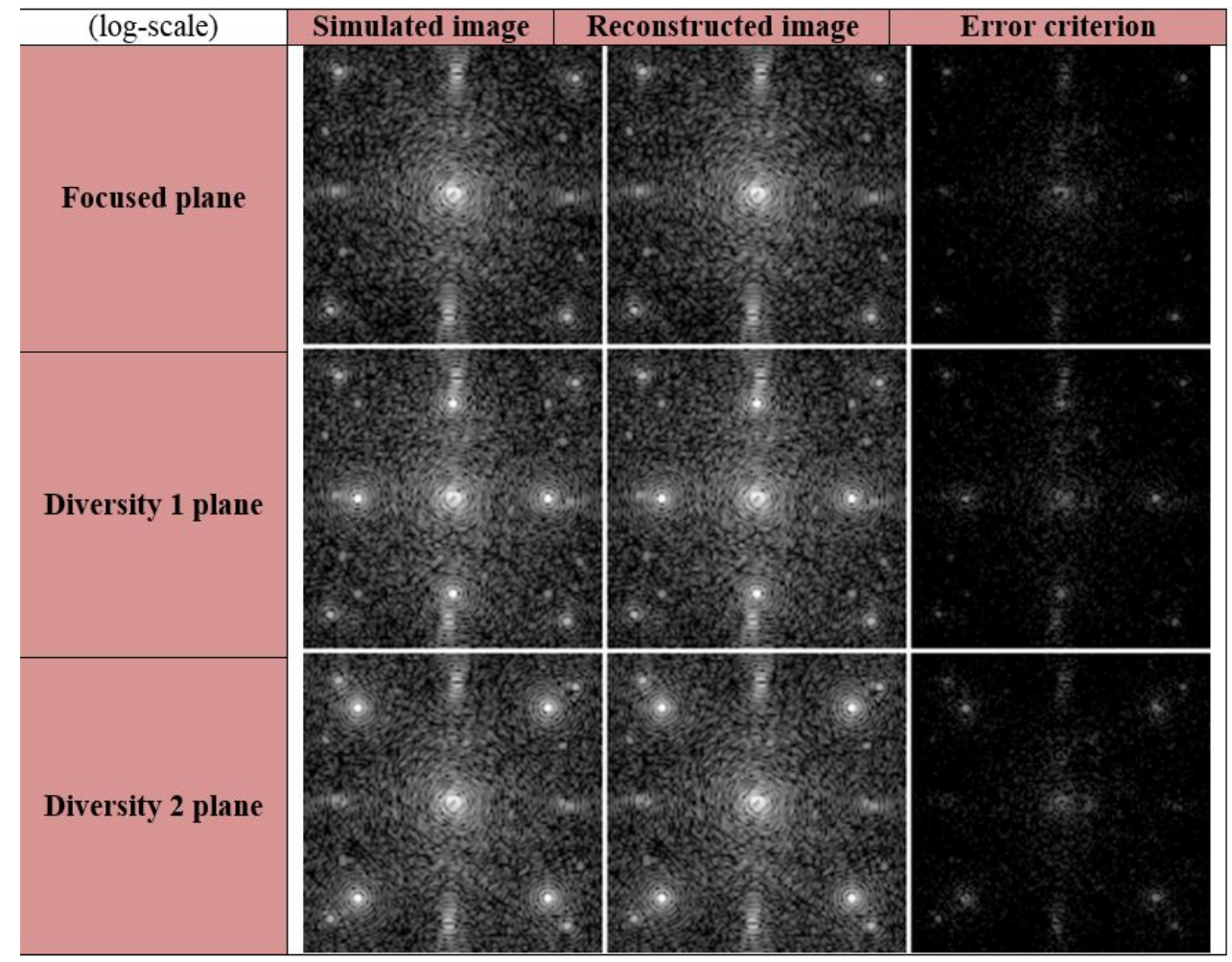

Figure 5. Simulated images (left column), images reconstructed by COFFEE (middle column), and difference (right column), for a waffle diversity. The effect of the waffle is to introduce four lateral peaks in the images. All images are shown in log scale.

Then, we study the influence of this waffle diversity on the reconstruction error. Figure 7 shows the reconstruction errors on the upstream phase, the upstream amplitude and the downstream phase as a function of the waffle amplitude. As with the usual low-order diversity, the errors go down when the diversity increases, but here a diversity of $50 \mathrm{~nm}$ is sufficient in order to obtain a nanometric accuracy both on the upstream and the downstream phases. 


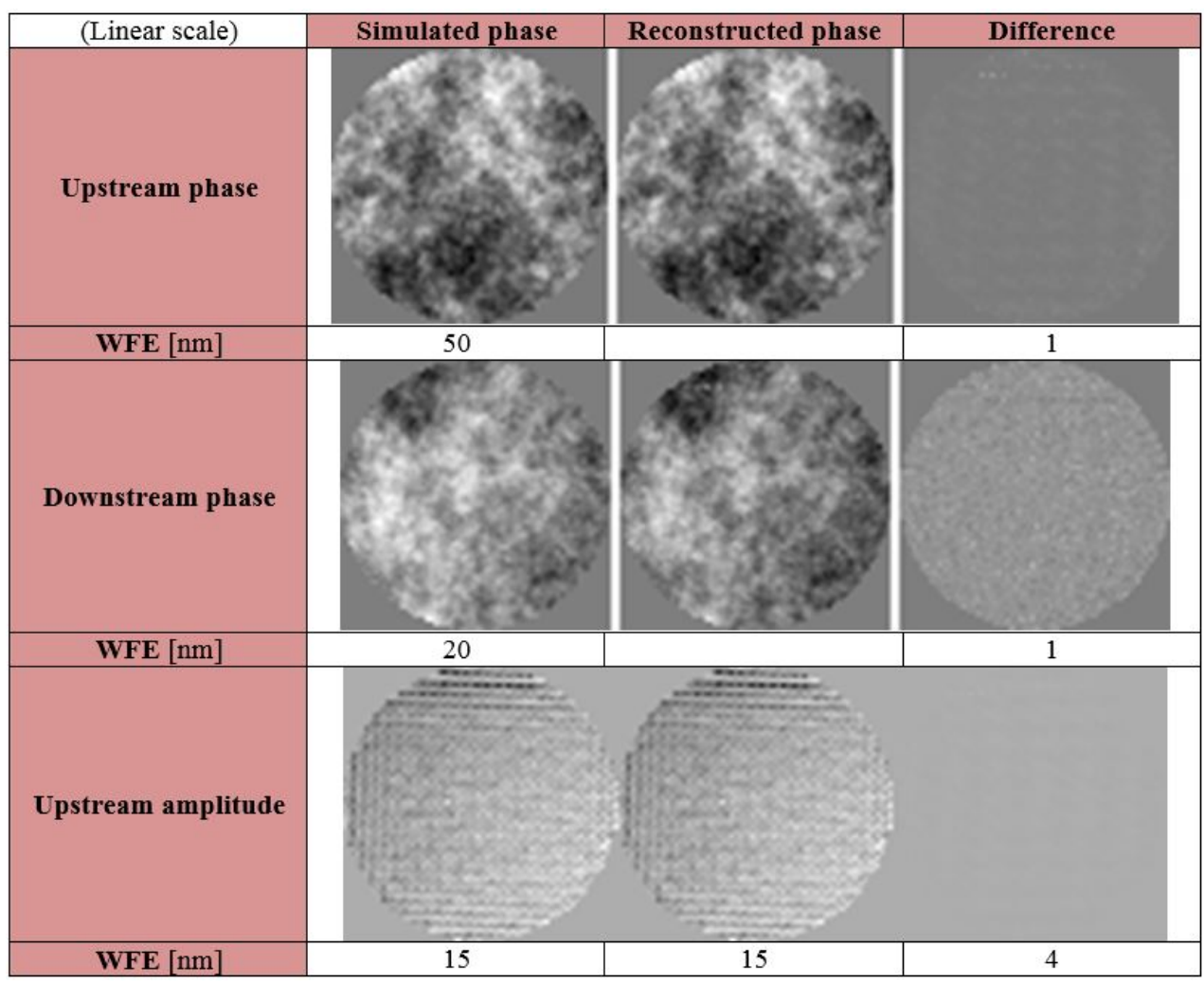

Figure 6. Simulated aberrations (left column), aberrations reconstructed by COFFEE (middle column), and difference (right column), for the upstream phase (top), the downstream phase (center) and the upstream amplitude (bottom). These reconstructions validate the use of waffle as a diversity phase.

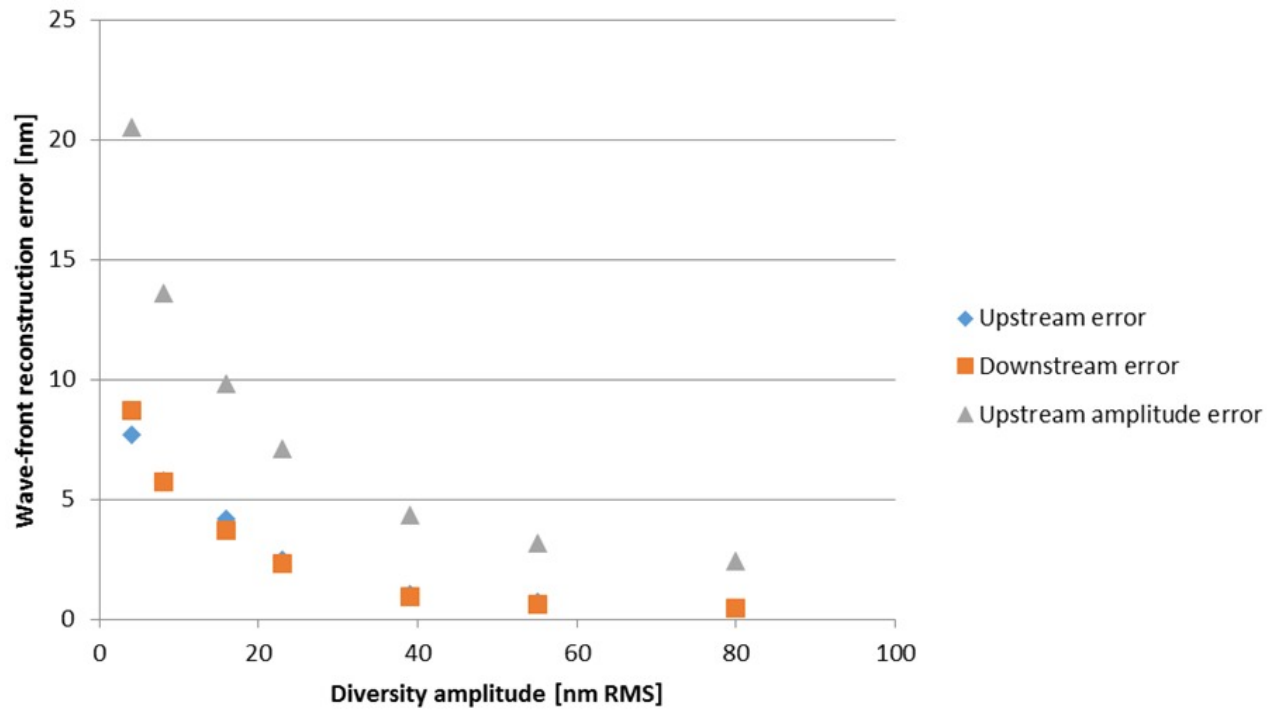

Figure 7. Optimization of the diversity phase for a waffle diversity: the RMS reconstruction errors of the upstream and downstream phases as well as of the upstream amplitude are plotted as a function of the amplitude of the waffle used for the two diversity images. 


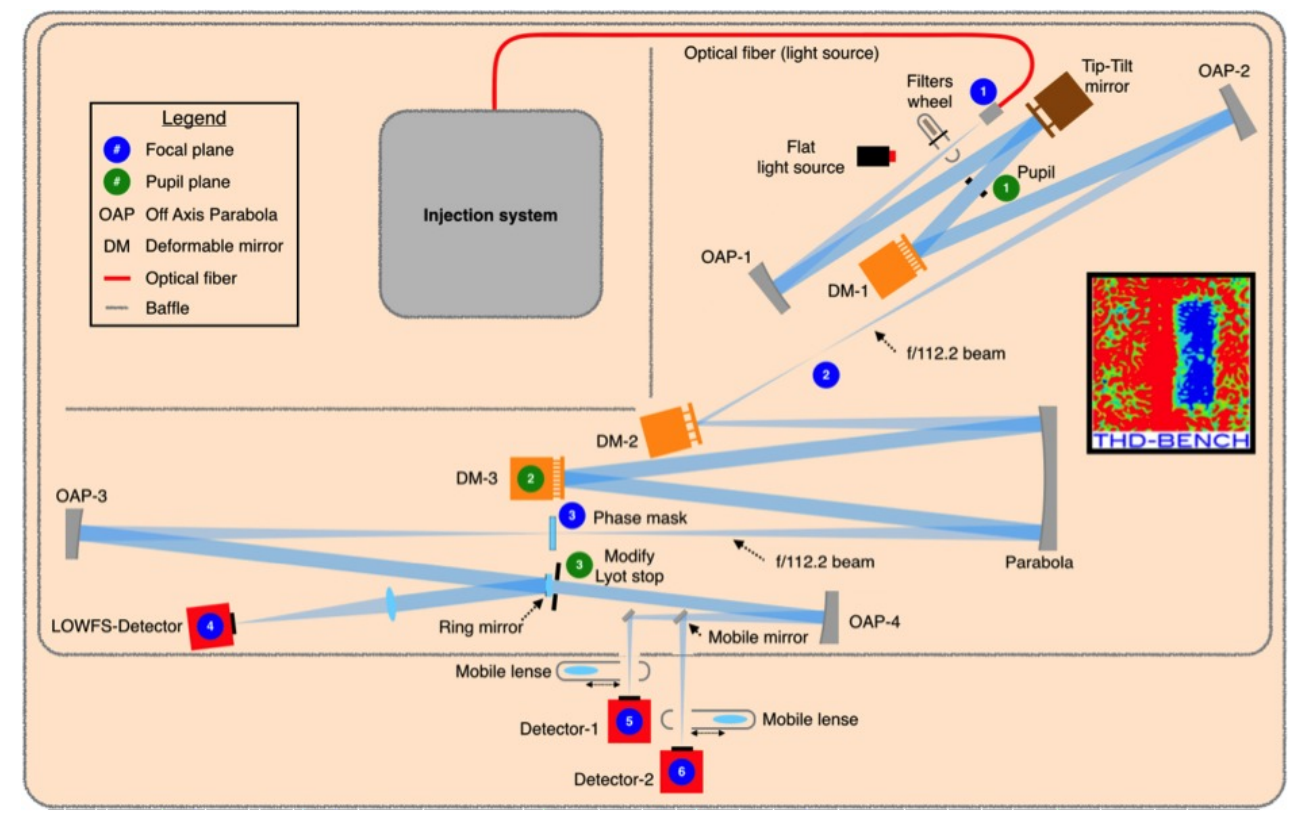

Figure 8. Schematics of the THD bench, including optics, components and beams.

\section{EXPERIMENTS OF COFFEE ON THE THD BENCH}

In this section, we validate COFFEE on the THD bench, which is a very high contrast imaging bench designed and built by Observatoire de Paris à Meudon, ${ }^{6,14}$ and shown on Fig. 8. Deformable mirror DM3 is a Boston Micro-Machines with $32 \times 32$ actuators in a pupil plane used to produce phase aberrations. DM1 and DM2 are placed away from a pupil plane in order to introduce amplitude aberrations. In the experiments described in this paper, DM1 is flattened and DM2 is replaced by a flat mirror. A preliminary work consisted in carefully calibrating the sampling of the detector and the diversity produced by the DM3. Indeed, such a mirror does not have a linear response, and phase diversity needs a precise calibration of the introduced diversity. In order to perform this calibration, we used classical phase diversity with the FQPM coronagraph removed, the following way: with DM3 flat, we recorded a high-SNR focal image of the point-source at $\lambda=783.25 \mathrm{~nm}$, then moved the camera by $16.5 \mathrm{~mm}$, which for an $\mathrm{F} \#$ of 60.6 translates into a peak-to-valley defocus of 4.51 radians, and recorded a diversity image. From these two images we used our phase diversity code to estimate the phase of the setup. Then we sent voltages to DM3 to produce, in theory, a focus, and we repeated the image measurements as well as the phase diversity estimation. The difference of the two estimated phases was then projected onto the first 210 Zernike polynomials in order to filter out noise. The results are shown on Fig. 9 and illustrate the diversity effectively produced by the DM and used in our subsequent, coronagraphic, reconstructions.

Starting from a nominal state of the bench with a contrast of the order of $210^{-8}$, we added a sine wave at $45^{\circ}$ with DM3, and we took 4 coronagraphic images, one focused and three with different levels of diversity. We then ran COFFEE to estimate the phase and the amplitude, using the diversity estimated in (non-coronagraphic) phase diversity as described above. Fig. 10 shows the recorded images and the images that COFFEE reconstructed at convergence. The spots at $45^{\circ}$ that are visible in the two first images are the focal-plane traces of the sine wave that we added on the DM. One notices that the images reconstructed by COFFEE are visually close to the recorded ones, which suggests that the minimization performed by COFFEE was effective.

Figure 11 shows the phase and the log-amplitude estimated by COFFEE. Although preliminary, this result shows that COFFEE has been able to rightfully assess that the sine wave was in phase and not in amplitude. The RMS value of the estimated phase is $4 \mathrm{~nm}$ RMS. The pattern in the log-amplitude can most likely be attributed to the DM itself. 


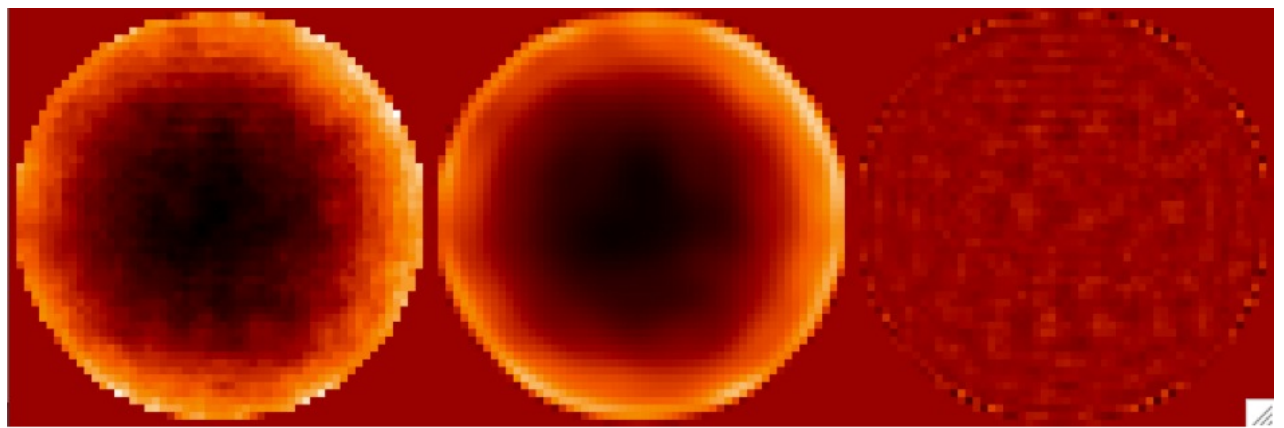

Figure 9. Left: diversity produced by the DM3, obtained as the difference of two classical phase diversity measurements. Middle: diversity on the left projected onto the first 210 Zernike polynomials to filter out noise. Right: difference of the two first images.

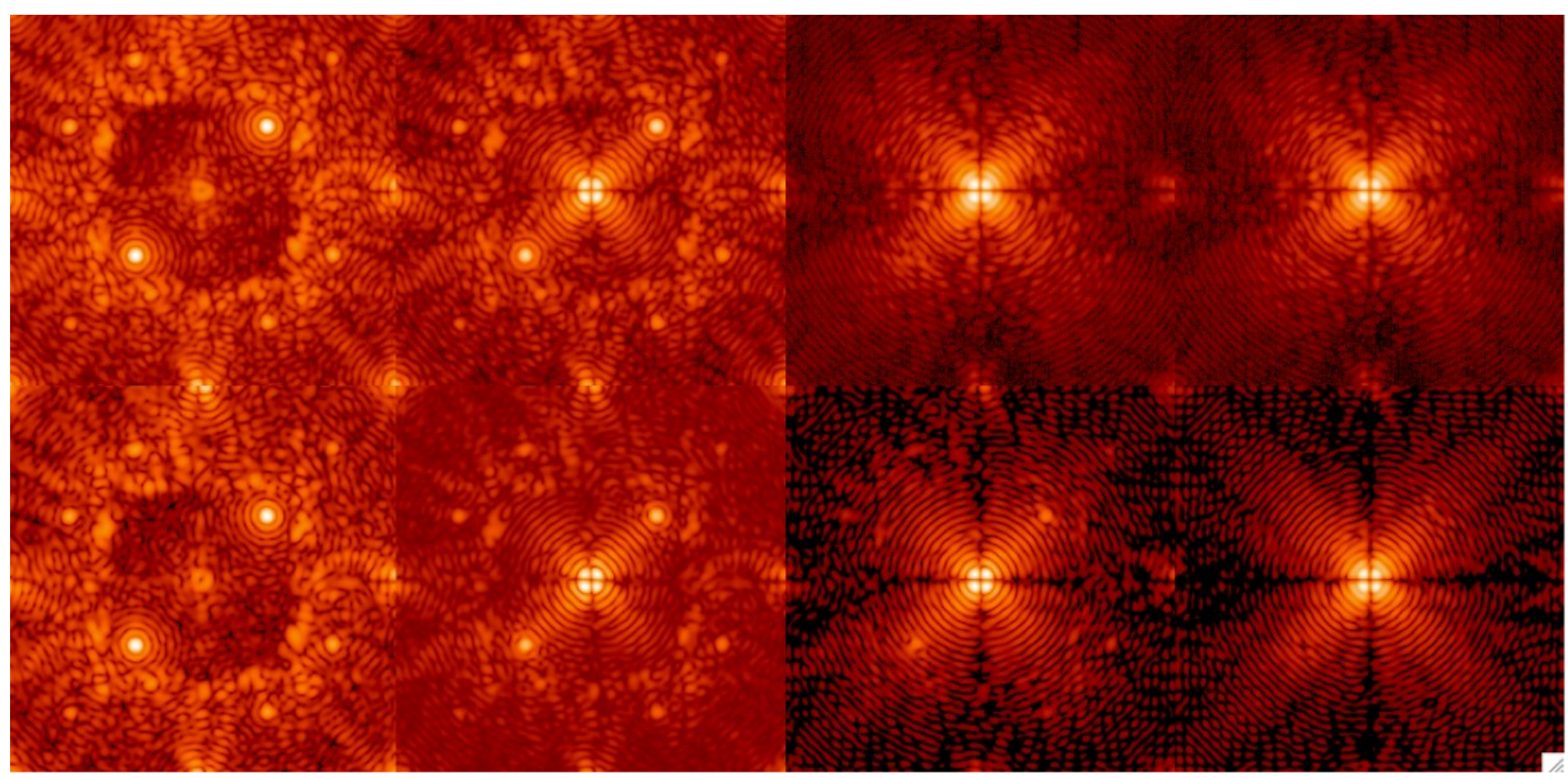

Figure 10. Top: focused image and diversity images recorded on the THD bench. Bottom: images reconstructed by COFFEE at convergence.
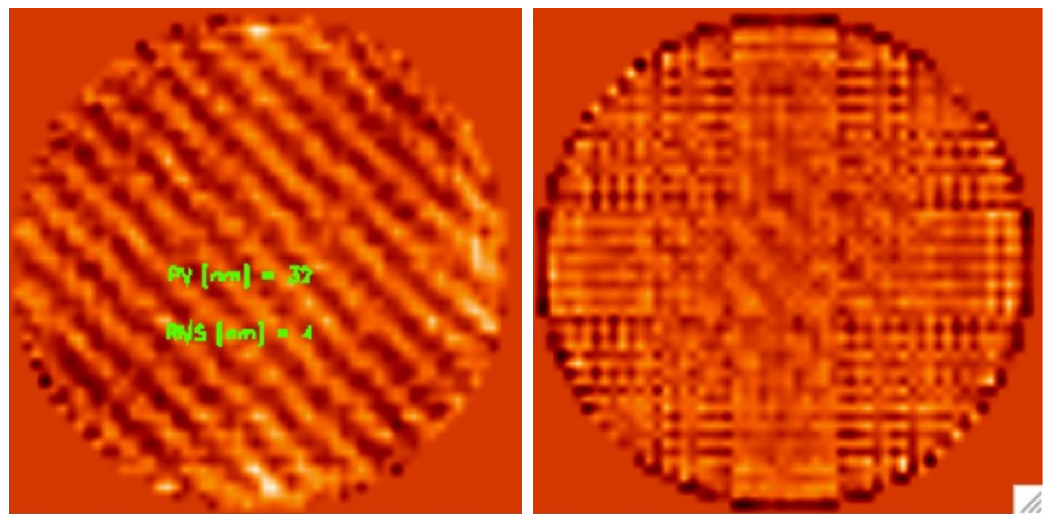

Figure 11. Field reconstructed by COFFEE: phase (left) and log-amplitude (right). 


\section{CONCLUSION}

In this communication, we have studied the extension of COFFEE to perform a simultaneous estimation of both phase and amplitude aberrations from three or more focal plane images. We have optimized the diversity phase in order to minimize the reconstruction error. We have also proposed and optimized a novel low-amplitude high-frequency diversity that should allow the phase-diverse images to still be used for science. Lastly, we have performed a preliminary experimental validation of COFFEE in the very high, space-like contrast conditions of the THD bench of Observatoire de Paris and showed that COFFEE is able to distinguish between phase and amplitude aberrations.

\section{REFERENCES}

[1] Sauvage, J.-F., Mugnier, L., Paul, B., and Villecroze, R., "Coronagraphic phase diversity: a simple focal plane sensor for high-contrast imaging," Opt. Lett. 37, 4808-4810 (Dec. 2012).

[2] Paul, B., Mugnier, L. M., Sauvage, J.-F., Dohlen, K., and Ferrari, M., "High-order myopic coronagraphic phase diversity (COFFEE) for wave-front control in high-contrast imaging systems," Opt. Express 21(26), 31751-31768 (2013).

[3] Paul, B., Sauvage, J.-F., Mugnier, L. M., Dohlen, K., Petit, C., Fusco, T., Mouillet, D., Beuzit, J.-L., and Ferrari, M., "Compensation of high-order quasi-static aberrations on SPHERE with the coronagraphic phase diversity COFFEE," Astron. Astrophys. 572, A32 (Nov. 2014).

[4] Bordé, P. J. and Traub, W. A., "high-contrast imaging from space: speckle nulling in a low-aberration regime," Astrophys. J. 638, 488-498 (Feb. 2006).

[5] Give'on, A., Belikov, R., Shaklan, S., and Kasdin, J., "Closed loop, DM diversity based, wave-front correction algorithm for high contrast imaging systems," Opt. Express 15 (2007).

[6] Baudoz, P., Boccaletti, A., Baudrand, J., and Rouan, D., "The self-coherent camera: a new tool for planet detection," in [Direct Imaging of Exoplanets: Science 85 Techniques. Proceedings of the International Astronomical Union Colloquium \# 200], Aime, C. and Vakili, F., eds., 1, 553-558, Cambridge University Press (2006).

[7] N'Diaye, M., Dohlen, K., Fusco, T., and Paul, B., "Calibration of quasi-static aberrations in exoplanet direct-imaging instruments with a zernike phase-mask sensor," Astron. Astrophys. 555 (July 2013).

[8] Mugnier, L. M., Fusco, T., and Conan, J.-M., "MISTRAL: a myopic edge-preserving image restoration method, with application to astronomical adaptive-optics-corrected long-exposure images.," J. Opt. Soc. Am. A 21, 1841-1854 (Oct. 2004).

[9] Thiébaut, É., "Optimization issues in blind deconvolution algorithms," in [Astronomical Data Analysis II], Starck, J.-L. and Murtagh, F. D., eds., 4847, 174-183, Proc. Soc. Photo-Opt. Instrum. Eng. (Dec. 2002).

[10] Mugnier, L. M., Blanc, A., and Idier, J., "Phase diversity: a technique for wave-front sensing and for diffraction-limited imaging," in [Advances in Imaging and Electron Physics], Hawkes, P., ed., 141, ch. 1, 1-76, Elsevier (2006).

[11] Meimon, S., Fusco, T., and Mugnier, L. M., "LIFT: a focal-plane wavefront sensor for real-time low-order sensing on faint sources," Opt. Lett. 35(18), 3036-3038 (2010).

[12] Paul, B., Sauvage, J.-F., and Mugnier, L. M., "Coronagraphic phase diversity: performance study and laboratory demonstration," Astron. Astrophys. 552 (Apr. 2013).

[13] Mazoyer, J., Baudoz, P., Galicher, R., Mas, M., and Rousset, G., "Estimation and correction of wavefront aberrations using the self-coherent camera: laboratory results," Astron. Astrophys. 557, A9 (Sept. 2013).

[14] Galicher, R., Baudoz, P., Delorme, J. R., Mazoyer, J., Rousset, G., Firminy, J., Boussaha, F., N'Diaye, M., Dohlen, K., and Caillat, A., "High contrast imaging on the THD bench: progress and upgrades," 9143, 91435A-91435A-11, Proc. Soc. Photo-Opt. Instrum. Eng. (2014). 

Office National d'Études et de Recherches Aérospatiales BP 72 - 29, Avenue de la Dicision Leclerc

92322 CHATILLON Cedex

Tél. : +33146734040 - Fax : +33146734141 http://www.onera.fr 